\title{
Virtual Reality and Electrodermal Activity to Support Mild Cognitive Impairment: A Systematic Literature Review
}

This paper was downloaded from TechRxiv (https://www.techrxiv.org).

\section{LICENSE}

CC BY 4.0

SUBMISSION DATE / POSTED DATE

08-02-2022 / 11-02-2022

\section{CITATION}

Patient, Rebecca (2022): Virtual Reality and Electrodermal Activity to Support Mild Cognitive Impairment: A Systematic Literature Review. TechRxiv. Preprint. https://doi.org/10.36227/techrxiv.19142279.v1

$\mathrm{DOI}$ 


\title{
Virtual Reality and Electrodermal Activity to Support Mild Cognitive Impairment: A Systematic Literature Review
}

\author{
Rebecca Patient, Fawaz Ghali, Hoshang Kolivand, William Hurst, and Nigel W. John
}

\begin{abstract}
This review explores mild cognitive impairment support, focusing on virtual reality (VR) applications coupled with electrodermal activity (EDA). A systematic review and meta-analysis using the Preferred Reporting Items for Systematic Reviews and Meta-Analyses (PRISMA) statement were performed from an original inquiry of 698 articles from 11 digital libraries. An independent review of selected studies assessed the risk of bias and obtained data. The meta-analysis included 44 studies, with a total number of 9099 participants in all the papers reviewed were split into (5200) cognitive or (2304) non-cognitive impairments. The proportion of cognitive versus non-cognitive had a first risk difference $(R D=\mathbf{0 . 3 2}$; p-value $=0.0001)$. The comparison of female to male participants showed $(O R=6.10 ; p$-value $=0.00001)$. Anxiety, depression, and stress conditions had the effect of (SMD = -0.39 ; $p$-value $=0.09$ ). In the analysis, the general lack of research restricts virtual reality and electrodermal activity to sustain mild cognitive impairments. Forty-five excluded papers applied data from pre-2017. Two studies with 46 participants had a result of $(S M D=0.60 ; p$-value $=0.31)$. Identifying VR and EDA-based studies investigating training and rehabilitation to support $\mathrm{MCl}$ symptoms in individuals.
\end{abstract}

Index Terms- Application, Electrodermal Activity, Mild Cognitive Impairment, Virtual Reality

\section{NOMENCLATURE}

$\mathrm{CBT}=$ Cognitive Behavioral Therapy, EDA=Electrodermal Activity, $\mathrm{MCl}=\mathrm{Mild}$ Cognitive Impairment, SCRs=Skin Conductance Responses, SLR= Systematic Literature Review, $\mathrm{SMD}=$ Standard Mean Deviation, VR=Virtual Reality, Inf = Infinite, $\mathrm{GC}=$ Google Cardboard

\section{Introduction}

$\mathrm{E}$ xploring mild cognitive impairment is a longstanding field within neurology and considered a transitional phase between normal cognition (normal aging process, slight changes in awareness and focus, such as missing a monthly

Rebecca Patient, Fawaz Ghali, Hoshang Kolivand are with Liverpool John Moores, Byrom Street, Liverpool, L3 3AF, Merseyside, United Kingdom, e-mail: r.patient@2014.ljmu.ac.uk, e-mail: F.Ghali@ljmu.ac.uk, e-mail: H.Kolivand@ljmu.ac.uk. William Hurst is with Information Technology Group, Leeuwenborch, Hollandseweg 1, 6706 KN, Netherlands, e-mail: will.hurst@wur.nl. Nigel W.John is with University of Chester, Parkgate Road, Chester, CH1 4BJ, United Kingdom, e-mail: n.john@chester.ac.uk payment) and MCI (small but more measurable deterioration in decision-making or memory, such as finding it hard to take care of all monthly bills or not knowing the date or time of year). At the time of writing this article, the entire world's population estimated to be living with a mental disorder was 50 million [1]. This number is predicted to triple to 131 million by 2050.5 to $20 \%$ of the UK population is diagnosed with MCI [2]. Therefore, detection and rehabilitation applications for individuals at a greater risk of developing MCI could be considered global priorities. MCI exists between the cognitive changes of normal ageing and mental illness. Individuals in this stage suffer from severe cognitive deficiencies but do not display functional impairments necessary to diagnose symptoms [3]. There is an increased rate of progression to those with a mental health disorder than those without MCI. Still, the cognitive decline of individuals with anxiety, stress and depression has the potential for improvement. Preceding systematic reviews from 2018-2020 regarding anxiety, stress, and depression uncovered promising effects on improving cognitive function between individuals with MCI and virtual reality therapy through COVID-19 [4]. In addition, ten recent review articles covering VR and EDA for supporting individuals with anxiety, stress and depression suffering from MCI indicate that developing technologies could improve symptoms using immersive reality, web-based, the Internet of Things (IoT), and artificial intelligence for assessment and therapy of individuals [5], [6], [7], [8], [9], [10], [11], [12], [13], [14].

The reviews were limited by accessing journal articles from a low number of databases and did not broadly encompass broader tools and applications. A solution for this problem and improving the review process is to apply keywords with a clear focus to reduce time and minimise the volume of data for improved results of existing research. For this reason, implementing an enhanced literature matrix for credibility, redundancy of information, and comparison of already research concepts were needed to supply an overview of the available position. This article provides a comprehensive review of VR and EDA techniques for supporting MCI symptoms in applications. This review has provided a foundation in research inspiring the development of virtual applications utilising hospitals and domestic simulated environments fitting a new field, keeping with other healthcare diagnoses and rehabilitation strategies (Cognitive Behavioural Therapy). These types of applications could assist save patients' lives through before-time 
diagnosis. For example, Prather et al.[7] and Liu et al. [11] argue that VR and EDA could improve MCI by implementing rehabilitation and training, to support improved learning outcomes [44]. Yet, Glegg et al. [13] disagree by stating that it could hurt participants and increase distraction, disrupting the cognitive impairment rehabilitation framework and resulting in poor outcomes. However, VR and EDA sustain healthcare staff and individuals to enhance health results. Current research determines the relationship between anxiety, depression, and stress with changes in physiological signals, an important aspect of wearable detection [31], [37], [63]. This research presents a framework for detecting anxiety, depression, and stress in a simulated environment by combining skin conductance and electrocardiography measurements. Individual skin conductance responses (SCRs) and heart rates are the primary sources of data in a skin conductance signal and the rate of occurrence related to psychological stimulation [15], [16], [24]. Challenges and functionality covering nearly the complete hypothetical field structure implied complex influences were needed to take on several elements that could influence the outcome and change of activities in any simulated environment [7], [69]. The volume of data accessible in this article validates the evolving position regarding the existing research. Indeed, qualitative cohort studies and literature reviews are valid methods of identifying key issues such as identifying study designs, method recommendations, and research questions [5], [10], [12], [17], [18].

\section{BACKGROUND}

Present research tends to apply quantitative data collection and analysing numerical meta-analysis. This type of approach provides the opportunity to widen the examination and frameworks for comparison to understand the changing aspects of VR better, EDA concerning randomised clinical trials [15], [17], [20], [25], [26], [27], [28]. For example, Glegg et al. [13] and Lyu et al.[28] are excluded from this SLR analysis because the investigations are limited to reviews, articles, and metaanalysis results pre-2018. Sources published in the past five years are an appropriate benchmark for the latest innovations, hypotheses, and approaches due to the fast pace of technological changes within the mixed reality domain [13], [19], [27], [29], [30]. When Wakefield et al. [8] considered the effectiveness of brief cognitive analytic therapy for anxiety and depression, a quasi-experimental case-control study, their study is similar to the theme demonstrated in the review. However, there are significant differences between cognitive analytic therapy and the two study groups. In that sense, the current research looks to identify triggers for MCI to support individuals. Whilst Moss [23] explored biofeedback-assisted relaxation training (BART) in 2020. BART is a widely applied biofeedback protocol in clinical practice from 1969 to the present for anxiety disorders, diabetes, headaches, and various other common medical and psychological conditions. However, this was a particular issue article and did not include any meta-data related to this research. The growth in VR and EDA in society, and the development of the healthcare industry, have seen the requirement for innovation in applications that can assess patients' needs. Identifying the most appropriate solution for their health is becoming more priority. With realtime assessment particularly notable in education, healthcare, sports and the commercial industry [86], [87], [91], [94]. A much-needed approach to detect MCI using health data with higher accuracy is a crucial topic that could lead to other health and emotional benefits [44], [60], [71]. Offering more information about MCI to individuals susceptible to anxiety, depression, and stress and psychological assessments indicates how beneficial MCI support detection is [9], [12], [24].

VR makes it possible to study such environments under controlled clinical conditions. Moreover, VR permits the time and cost-effective remoteness and revision of variables (activities), unrealistic in the real world. VR and EDA are becoming evolving technologies that enable individuals to obtain physiological data outside of the real world by establishing a simulated environment-allowing individuals to detect and monitor their health and develop experiences (similar to how they would do so in the physical world) to identify and improve MCI. Indeed VR and EDA challenge individuals' awareness and assessment of their health and simulated environment. Simultaneously, EDA collates individual data about heart rate whilst completing tasks [87]. Therefore, the integration of VR and EDA frameworks are based upon simulated computer graphics, generating virtual elements that individuals can understand and control through different input devices (e.g. Google Cardboard, Oculus Rift, and Quest) combined with output device EDA (e.g. Fitbit or other wearable technology) [31], [37], [63]. Instead of human senses being optimal for VR, haptics and VR technology are developed and adapted to human senses sensors (hearing, vision, and touch) that may be applied and promoted for individual engagement through simulation, enabling the framework to identify and assist MCI symptoms. Individuals naturally prefer a multimodal approach for a higher level of interaction [31], [57], [67], [83]. Yet, challenges include i) cost, ii) storage of data, iii) participant sample size and d) requirements of users, as identified in the following studies [9], [12], [13], [23], [24], [26], [28], [29], [30], [33], [37], [38]. Regarding point (iii), no minimal standardisation of 30 participants was implemented in 91 studies for increasing the validity and reliability of the research conducted, thus not facilitating confidence in studies performed. Therefore, any potential benefits may be lessened because of the smaller sample size. Other potential challenges iv) hardware, v) software integration and vi) training new skills were highlighted by Hsieh et al. [32] and Gold et al. [42] in 2018, who identified innovation in technology with these frameworks; they should be implemented in the healthcare industry. This type of implementation is the financial implication of hardware, software integration, and connection to hospital databases and equipment for staff and individuals, making it simpler to utilise and support MCI and long-term application heath. Moreover, training recruits and patients is a potential challenge as we advance. However, Gold [42] also stated it was more important to maintain standard hygiene processes to reduce patient infection and share technology [34], [36]. Whereas Weech et al.[18], in 2019, was more concerned 
with the measurement authenticity and cross-validation of the measurement entity. The author found a restricted number of datasets related to human research and VR that became when comparing results [18].

Furthermore, a ubiquitous deficiency in data science within many surveys were assessed [35]. Studies involving MCI have concentrated on awareness for adults or those with MCI. However, there has been an increase of patient-centric support groups who later become representatives of healthcare teams who can then voice their own opinions about treatment [7]. More recently, additional application-based support studies have been conducted, including meta-analysis and a pilotstudy to evaluate the impact of an innovative combined physical activity and cognitive training based on VR in mild cognitive impairment patients [5], [20], [23]. Consequently, the aim of this analysis is to 1) encapsulate journals using applications in VR and EDA by conducting a thorough search applying scientific and biomedical literature databases, 2) understand the effect of using VR and EDA applications on improving capabilities between people with mild cognitive abilities, 3 ) explore the effects of multi-model application-based support integration compared to single component support detection and 4) present the findings and offer future directions for this work.

\section{Mild Cognitive IMPAIRMENT}

The Alzheimer's Society defines MCI as a condition where an individual has minor issues with cognition [44]. These lesser issues are mental aptitude inspiring decision making and memory, which would be worse than individuals expected to be healthy. Examples include forgetting or repeating the same question, not remembering the names of friends, or misplacing objects. Navigation issues when not quick thinking of how they reached a location or regain the knowledge to where they were previously. Those diagnosed with severe conditions are more likely to suffer from symptoms than those who do not have MCI. Severe cognitive impairments signify individuals losing the aptitude to comprehend the meaning of something with the facility to write, talk or use fundamental techniques such as a tv remote or a kettle. The main reasoning is not adept at living independently. Shankle [1] stated that the underlying causes of MCI are anxiety, stress, and depression because of various medications, such as sleeping tablets, antidepressants, and pain medication. Conditions are curable, mainly when cognitive memory disorders are detected early through annual cognition monitoring after age 50 years old [1]. MCI, however, may appear and vanish, unlike the diseases themselves. In particular, healthcare professionals may have trouble identifying an individual's condition. Individuals have difficulty remembering, understanding, concentrating, or being indecisive, changing their daily experiences. In addition, friends and family could observe confusion, anxiety, or behavioural changes, such as speech or interaction challenges.

\section{A. Search Strategy \& Experimental Study Selection}

Recent developments in VR technologies, signal processing for EDA, and ML algorithms could provide longterm solutions to support MCI [2], [3], [4], [6]. In addition, these tools could improve diagnosis and therapy and contribute to the disorder's detection [9], [12]. Therefore, the associated literature review string, shown in Table I, was applied based on keywords relating to these technologies. The systematic review in Figure 1 was performed according to Revman Cochrane Handbook and Revman 5.4.1 software [46]. In conjunction with PRISMA Declaration guidelines [45]. A literature review was mapped out to highlight the research's central concept similar to other papers within the field [5], [6], [7], [13]. The analysis in this SLR relied upon the most important primary sources of the article matrix investigation of existing research and the evaluation of meta-data to compare results.

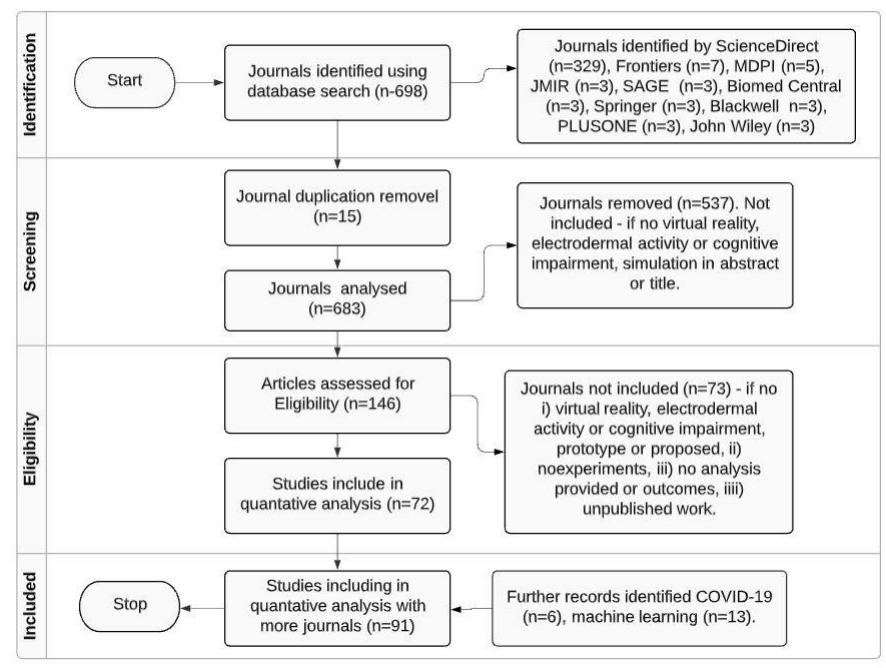

Fig. 1. Journal Search Strategy

TABLE I

KEYWORD SEARCH METHOD

\begin{tabular}{ll}
\hline Keyword Search & Query String \\
\hline Participants, & ("COVID-19" OR "Corona Virus" OR "anxiety" OR \\
Intervention, & "Stress" OR "Depression" OR "Applications" OR \\
Detection, & "Support", "Cognitive" OR "Impairment" OR "Virtual \\
Comparison \& & $\begin{array}{l}\text { Reality" OR "Electrodermal Sensors" OR "Cognitive } \\
\text { Outcome }\end{array}$ \\
$\begin{array}{l}\text { Impairment" OR" Machine Learning" OR "Metadata" } \\
\text { or "bio-signals) }\end{array}$ \\
\hline
\end{tabular}

TABLE II

PRIMARY SELECTION METHOD

\begin{tabular}{lc}
\hline METHOD & Number of Papers \\
\hline Eligibility & 146 \\
Focus on meta-analysis & 72 \\
Exclusion from research & 73 \\
Additional Papers analyzing COVID-19 \& ML & 19 \\
\hline Total Article Total & $\mathrm{N}=92$ \\
\hline
\end{tabular}

The analysis in this SLR relied upon the primary sources of existing research and the evaluation of meta-data to compare results. This type of review has been widely applied to systematically research fields to locate a central body of works. Using a literature search process, it is possible to systematically analyse and classify large volumes of peer-reviewed literature in a specific research domain [10], [11], [14]. Therefore, this literature search was undertaken to connect the concepts of VR, $\mathrm{EDA}$, and MCI. The literature search analyses studies explicitly 
dedicated to VR applications for a more generalised outlook, detecting bio-signals using ML techniques from gathering EDA. The total number of articles analysed was 91, as displayed in Figure 1. Table II was applied to reduce the number of articles. At the same time, Table III demonstrates a series of inclusion and exclusion criteria established to refine the literature search results and reach the papers' final selection. The standards applied to include or exclude publications are stated.

TABLE III

INCLUSION \& EXCLUSION CRITERIA

\begin{tabular}{|c|c|c|}
\hline $\mathrm{Nr}$ & Inclusion & Exclusion \\
\hline SQ1: Article Search & $\begin{array}{l}\text { Application, Design, } \\
\text { Experiments, Meta-data, } \\
\text { and ML. }\end{array}$ & No Exclusion \\
\hline $\begin{array}{l}\text { SQ2: Outside } \\
\text { influence }\end{array}$ & COVID-19 & No Exclusion \\
\hline SQ3: Participants & Population, Gender & No Exclusion \\
\hline SQ4: Studies & $\begin{array}{l}\text { Language, Randomised } \\
\text { Controlled Trials, Case } \\
\text { Study }\end{array}$ & $\begin{array}{l}\text { Non-randomised, } \\
\text { Uncontrolled Data }\end{array}$ \\
\hline $\begin{array}{l}\text { SQ5: Intervention/ } \\
\text { Detection }\end{array}$ & $\begin{array}{l}\text { Training, Learning, } \\
\text { Rehabilitation }\end{array}$ & $\begin{array}{l}\text { Studies Including Non- } \\
\text { Relevant Disorders }\end{array}$ \\
\hline $\begin{array}{l}\text { SQ6: Outcome } \\
\text { Measures }\end{array}$ & $\begin{array}{l}\text { Blood Pressure, Heart } \\
\text { Rate, Bio-signals }\end{array}$ & $\begin{array}{l}\text { Any Other Physical Or } \\
\text { Non-Physical Limits }\end{array}$ \\
\hline
\end{tabular}

TABLE IV

KEYWORD SEARCH METHOD

\begin{tabular}{ll}
\hline Database & Papers \\
\hline ScienceDirect & $\mathrm{n}=321$ \\
IEEE & $\mathrm{n}=329$ \\
Frontiers Media SA. & $\mathrm{n}=7$ \\
MDPI & $\mathrm{n}=5$ \\
BioMed Central Ltd. SAGE Publications. PLOS & $\mathrm{n}=21$ \\
ONE. Blackwell Publishing Inc, Springer, JMIR & \\
Publications, John Wiley and Sons Ltd & \\
\hline Raw Data Total & $\mathrm{N}=698$ \\
\hline
\end{tabular}

\section{B. Experimental Study Selection}

The keywords searched in all digital databases indicated in Table IV, and a total of 698 papers generated in the search. Fifteen duplicates were eliminated from the various databases. 146 papers were also taken out as the research criteria did not meet the eligibility requirement, and it was necessary only to select articles including VR, EDA, MCI, ML, and COVID-19. 74 articles were removed after further examination and reading the full content. 19 additional articles were included because they discussed COVID-19 and ML. A total of 91 articles were then investigated in this survey and placed in a literature matrix for comparative analysis (however, VR, MCI, and ML were not combined in all 91 studies included in Table II).

\section{Journal Classification Categories}

The literature analysis studied in this review was identified using different criteria to identify similarities in fields, disorders, and possible experiments [45], [46]. Table V shows that articles selected within the search can be classified into three main categories. The first category to be categorised was VR, which originates from different areas analysing simulations in terms of navigation. Various technologies were involved in the studies (e.g. HTC Vive, Oculus Rift, and HoloLens). In contrast, this research aims to apply the latest innovations (e.g., the Oculus Quest 2 and Unity) for therapy and rehabilitation. The second classified category was selected by detecting MCI symptoms (anxiety, stress, and depression). The 91 primary studies applied as input included in this research are listed in Table V. Whereas Table VI (Outcomes of MCI Support vs Applications) illustrates the statistical methods applied in existing research. The publication year of these studies ranges from the years 2018 to mid-2021. It also includes a list of all journals used in this survey, grouped by the classification method. Logically, there are some replicated articles between the three columns. There is no separating of the content in most of them. For example, the design of an application requires VR and EDA to be processed. ML and EDA are to be administered concurrently. It should also be noted that where duplication of reference numbers in all tables within this document, a further search was implemented.

TABLE V

PRIMARY STUDIES APPLIED AS INPUT

\begin{tabular}{ll}
\hline Concept & Studies \\
\hline VR & {$[6],[5],[7],[12],[13],[15],[17],[18],[20],[21],[22],[23]$,} \\
& {$[26],[27],[30],[31],[32],[33],[34],[36],[35],[37],[38]$,} \\
& {$[41],[42],[43],[47],[49],[50],[54],[57],[59],[60],[61]$} \\
EDA & {$[2],[3],[8],[9],[11],[12],[16],[20],[25],[26],[27],[28]$,} \\
& {$[29],[30],[31],[32],[33],[34],[35],[36],[37],[38],[42]$} \\
MCI & {$[2],[3],[8],[9],[11],[12],[20],[25],[26],[27],[28],[29]$,} \\
& {$[30],[31],[32],[33],[34],[35],[36],[37],[38],[39],[42]$} \\
COVID-19 & {$[2],[3],[4],[6],[27],[34],[38],[69],[80],[71],[92]$} \\
\hline
\end{tabular}

TABLE VI

OUTCOMES OF MCI SUPPORT VS APPLICATIONS

\begin{tabular}{|c|c|c|c|}
\hline Outcome & Studies & Participants & Statistical Method \\
\hline $\begin{array}{l}\text { 1.1 Proportion of } \\
\text { Cognitive vs Non- } \\
\text { Cognitive }\end{array}$ & 44 & 18198 & $\begin{array}{l}\text { Risk Difference (M- } \\
\text { H, Fixed, 95\% MCI) }\end{array}$ \\
\hline $\begin{array}{l}\text { 1.2 Comparison of } \\
\text { Female vs Male } \\
\text { Participants }\end{array}$ & 26 & 8858 & $\begin{array}{l}\text { Odds Ratio (M-H, } \\
\text { Random, 95\% MCI) }\end{array}$ \\
\hline $\begin{array}{l}\text { 1.3 Anxiety, } \\
\text { Depression \& Stress }\end{array}$ & 7 & 3195 & $\begin{array}{l}\text { Std. Mean Difference (IV, } \\
\text { Random, 95\% CI) }\end{array}$ \\
\hline 1.4 VR and EDA & 2 & 91 & $\begin{array}{l}\text { Std. Mean Difference (IV, } \\
\text { Random, 95\% MCI) }\end{array}$ \\
\hline
\end{tabular}

\section{MCl Support vs Application in Meta-Analysis}

This section will discuss the three main concepts combined in this systematic literature review: MCI, VR, and EDA. Bringing these concepts together allows for an analysis of applications in VR and EDA for MCI support, shown in Table VI. Three different statistical methods were applied using Risk, Std Mean Difference, and Odds Ratio, which indicated an average $95 \%$ confidence interval. Although, differences were found in the effect estimate through authors applying different technologies and experimental variables, such as utilising randomised control/clinical studies, case study or various numbers of participants without a comparative analysis [4], [72], [73], [77]. Figure 3 and Figure 4 indicate, from 91 studies, 
that the total number of participants with either cognitive or non-cognitive was 98578 with an effect estimate using an Odds ratio of $2.98[0.69,12.884]$. The diamond demonstrates the point estimate, which in this case, favours non-cognitive participants. Heterogeneity of the journals was: Tau2 $=2.30$; Chi2 $=1235.45, \mathrm{df}=31(\mathrm{p}$-value $<0.00001) ; \mathrm{I} 2=97 \%$. The I2 should be below $50 \%$, which is not the case from the review, which means there could be inconsistencies within the research. The test for overall effect: $Z=1.46(p$-value $=0.14)$. The percentile was calculated using $100 \times \operatorname{Pr}(Z<1.46)=100 \times 0.9279=92.79 \%$ shown in Figure $2[45$, 46].

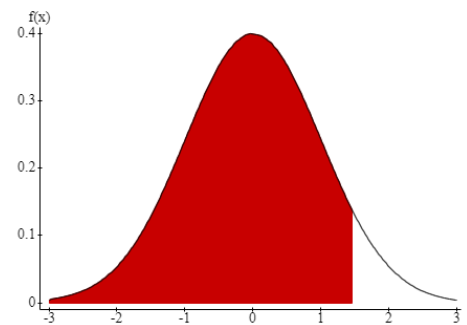

Fig. 2. The 93-th percentile is associated with the $\mathrm{Z}$-Score $-\mathrm{z}=1.46$

\section{E. Risk of Bias in Studies}

A three-tier color system based on Cochrane Reviews was implemented in Figure 3, where red $=$ high risk; yellow with question mark $=$ unclear; green $=$ low risk. The risk of bias is placed into sections: A) Random sequence generation (selection bias), B) Allocation concealment (selection bias), C) Blinding of participants and personnel (performance), D) Blinding of outcome assessment (detection bias), E) Incomplete outcome data (attrition bias), F) Selective reporting (reporting bias), and G) Other bias [46]. All experiments combined two of the three colors except for Wakefield et al. and Jungmann et al., and Parong et al., which showed high risk [52], [64], [82]. Wakefield et al. deployed a quasi-experimental case-control study, conducted a single, improving access to Psychological Therapies (IAPT) service, and used sessional outcome monitoring. Propensity score matching was used to derive equivalent CAT $(\mathrm{N}=76)$ and $\mathrm{CBT}(\mathrm{N}=73)$ samples through matching intake characteristics. Longitudinal multilevel modelling (LMLM) compared patterns of symptomatic change over time between the two therapies [8]. Jungmann et al. undertook The Short Health Anxiety Inventory (SHAI), Cyberchondria Severity Scale (CSS15) [38]. All other studies showed a combination of low risk in the generation of the randomisation sequence. All were at high risk or yellow for unclear risks of bias, and three studies were in the blinding of outcome assessments.

\section{F. Results}

No studies had a low risk of bias in all assessed criteria. The total heterogeneity was Chi2 $=2872954.40, \mathrm{df}=43$ (p-value $<$ 0.00001 ); I $2=100 \%$ for Outcome 1.1 Proportion of Cognitive vs Non-Cognitive. Outcome 1.2 Comparison of Female vs Male Participants has a total heterogeneity: Chi2 $=804.12, \mathrm{df}=25$ (p-value $<0.00001$ ); I2 =97\%. Whereas Outcome 1.3 Anxiety, Depression and Stress show a total Heterogeneity: Tau2 $=0.31$; Chi2 $=152.60, \mathrm{df}=6(\mathrm{p}$-value $<0.00001) ; \mathrm{I} 2=96 \%$. Outcome $1.4 \mathrm{VR}$ and EDA showed a total heterogeneity: Tau2 $=0.59$;
Chi2 $=7.06, \mathrm{df}=1$ (p-value $=0.008) ; \mathrm{I} 2=86 \%$ This does indicate inconsistency in participants and the results for VR, EDA and MCI. The studies observed significant differences between the participants and disorders from post-traumatic stress, cancer, arachnophobia concerning interventions, measurement of outcomes, and requirements within a metaanalysis. Figure 3, shows a funnel plot of the 44 studies included in the meta-analysis. Figure 4, favours more noncognitive studies, whereas the funnel plot is relatively symmetrical. Many studies fall outside the triangle, indicating heterogeneity in the strength of the association between cognitive and non-cognitive was also present in studies categorised (for example COVID-19 ([6], [26]), Challenges ([7], [13], [17]) and Application ([5], [10], [12], [20]).

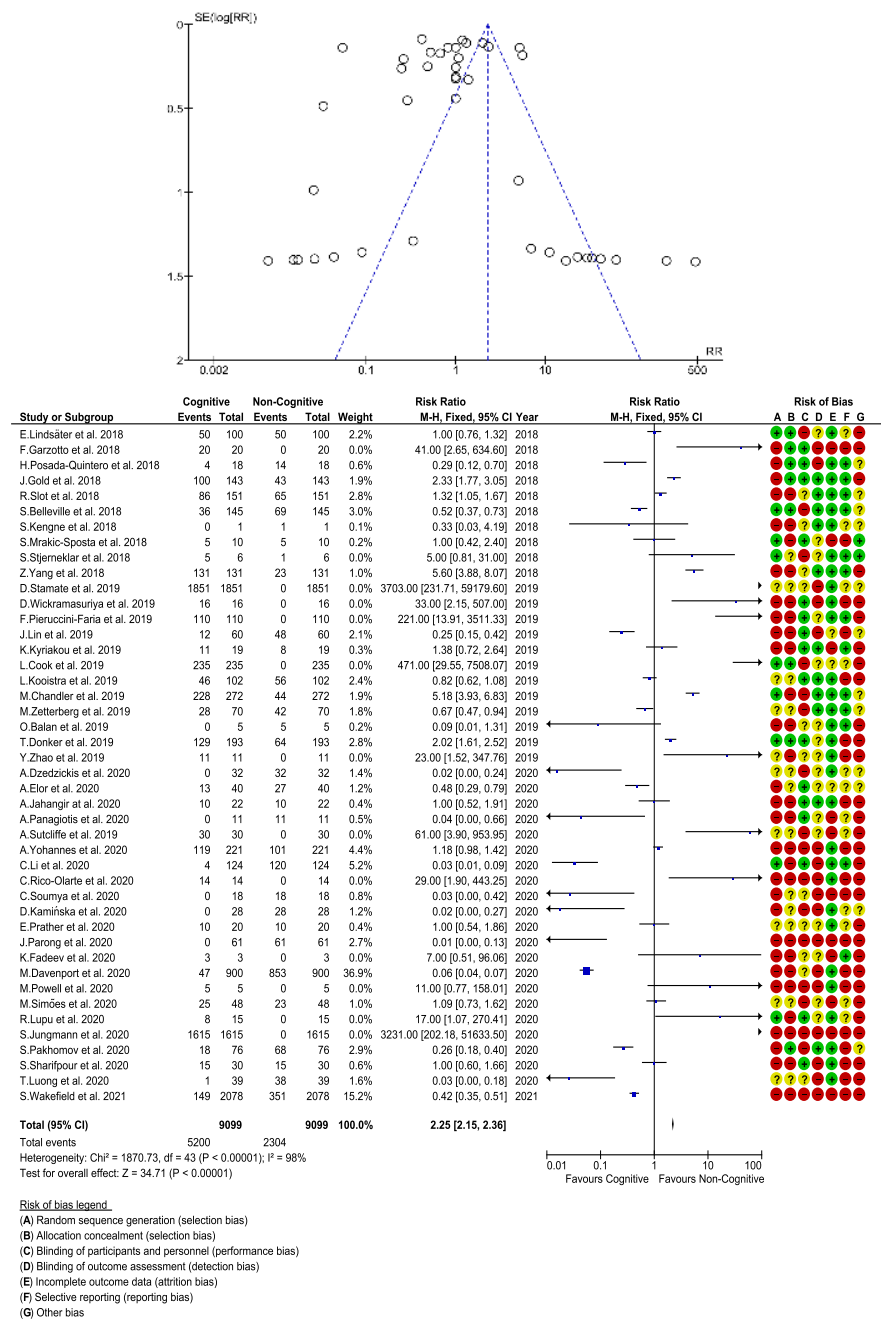

Figure 3. Funnel and Forest plot of comparison with Risk of Bias: 1 Cognitive Impairment Support vs Applications, outcome: 1.1 Proportion of Cognitive vs Non-Cognitive

TABLE VII

APPLICATIONS IN VR AND EDA BY YEAR

\begin{tabular}{lll}
\hline Year & Application & Total \\
\hline 2018 & {$[22],[32],[35],[42],[72]$} & 5 \\
2019 & {$[11],[24],[20],[47],[89]$} & 5 \\
2020 & {$[4],[7],[27],[28],[54],[55],[57],[63]$,} & 13 \\
& {$[64],[65],[73],[77],[91]$} & \\
\hline
\end{tabular}




\section{G. Application in VR \& EDA}

VR is becoming a revolutionary way for integrating computers with IoT. The equipment eliminates customary partition among devices and users. Communication is becoming more intuitive and straightforward regarding the information and data provided and engagement. The headsets listed in Table VIII are the most commonly available and incorporate an audio and visual display, hand tracking, position, cameras, sensors for navigation, and hands-on interface tools. It is now possible to create real-world environments within virtual worlds that allow users to visualise, touch, manage and hear [45], [87]. HP Reverb G2 is worth considering because it is eye-tracking and monitors heart rate, requiring a USB connection to a PC [56]. The Oculus Quest 2 used a wireless connection to a mobile device for an initial set-up and was released in August 2021 and (at the time of writing this article) is less expensive.

TABLE VIII COMPARISON OF THE LATEST VR HEADSETS

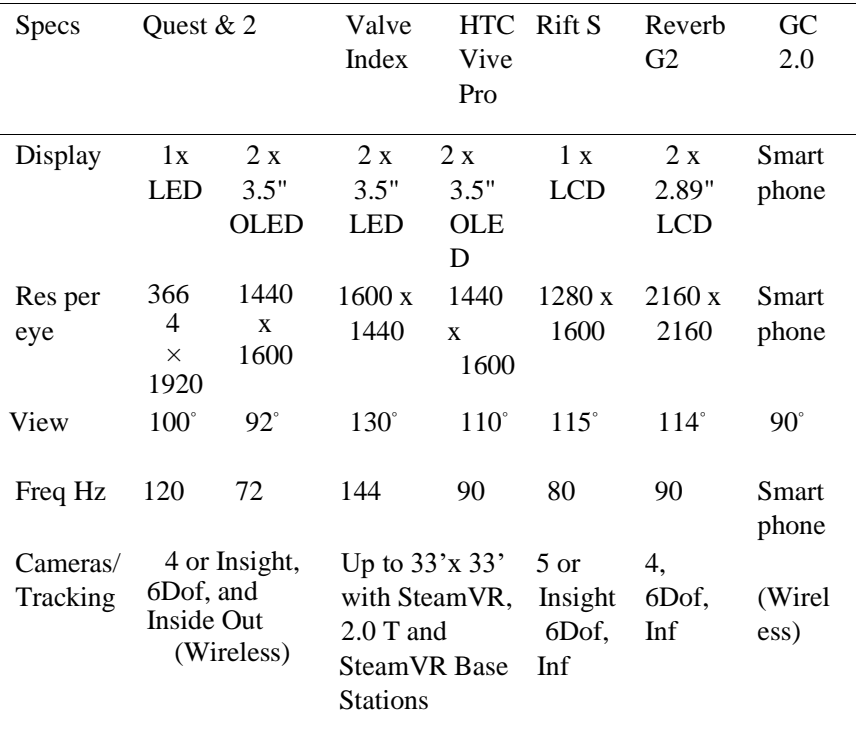

Innovation in EDA is targeted prominently towards observing individual physiological parameters, such as EDA, heart rate, and sweat glands in everyday life over timeconsuming intervals. Uninterrupted monitoring of such parameters facilitates predicting mental states and stress, intending to deliver responses to enhance the results [31], [37], [63]. However, the utilisation of these applications in the real world is still convoluted and susceptible to error handling issues because of poor quality data collection, which influences objects' existence in this survey an ML approach to detect features automatically using EDA can be gathered in VR and real-world over time [51], [53]. Intrinsic active modifications permit various measurements to be captured using several biosignals. These sensors provide time-altering indicators for the human brain's cognitive state through physical and psychological accuracy [16], [19]. The EDA has bio-signals that include skin conductance and changes in acceleration, pressure, sound, flow, and temperature [8], [50], [71]. Accurate monitoring of EDA provides an individual's emotional, cognitive, and mental state framework. Therefore, the biosignals approach should be considered, especially with the applications deployed for available applications.

\section{Discussion of Results}

This research aims to establish a systematic review of literature in applying VR and EDA within the context of supporting MCI. Table V and VI summarise the obtained results from these; it appears there is a growing interest in VR applications. Researchers had submitted 92 papers for publication in the last decade, ranging from education to the medical and commercial industries. Significant technological advances are ever-growing in VR headsets and EDA sensors. The medical and education field should dedicate more vigorous analysis to the assessment, diagnosis, and rehabilitation stages to develop enhanced health applications. VR is the most addressed aspect, as 34 articles out of 92 discuss technical significance in-depth, shown in Table VIII. At the same time, EDA (23) and MCI (23) are further research and development topics. There is a need for machine learning, a multiple parameter framework with implementation. 19 additional articles cover ML and Covid-19 with VR analysis. Viewpoints from research and medical fields are required. 23 articles investigating applications in VR and EDA submitted having frameworks for various health conditions from strokes, Parkinson's, Alzheimer's, dementia but not specifically for MCI symptoms.

In that follows current advances in the field for each topic within Table VI, and the deep analysis of the articles analysed in Figure 3. Involvement of humans for the goals of training and rehabilitation Table VIII summarises the most recent advancements in MCI virtual reality. Within the 91 publications, virtual reality is an example application; On Shooting Stars: Comparing CAVE and HMD Immersive Virtual Reality Exergaming for Adults with Mixed Ability. OpenButterfly: Multimodal Rehabilitation Analysis of Immersive Virtual Reality for Physical Therapy, and Effectiveness of self-guided app-based virtual reality cognitive behaviour therapy for acrophobia: a randomised clinical trial [67], [70], [86] were among the studies included. However, these studies are from 2019 to 2020, whereas other research is from 2018 to 2020; therefore, the impact factor may be lower than the number of other publications mentioned in that span, making it less critical.

The application of VR and EDA for the significance of MCI

support could emerge as a real-time individual user-driven system. The framework is not necessarily required to be a general assessment for diagnosis but more of a practical assessment of a user implementing basic activities such as walking, standing, and sitting down. Additional innovation could include basic user input to gauge user interaction and cognition. Therefore assessment, diagnosis, and rehabilitation for the definition of MCI symptoms (confusion, inability to solve problems, losing train of thought, and or repeating themselves) remain a critical point regarding short-term memory issues and effectiveness to retrain the mind. For instance, counseling and assessments are still essential methods in cognitive behavioural therapy for post-stroke depression. In 
high-intensity VR exergaming, they were looking for and recognising links. Efficacy and durability are two factors that can influence mental health. However, in studies such as [39], [52], [55], [71], training and psychosocial intervention are suggested as methods to assist in preventing future problems.

According to this field, individuals with MCI symptoms may benefit from cognitive therapy, training, and physical activity. For example, studies using virtual reality and EDA such by Johnston et al. and Panagiotis et al.. Exploring pedagogical underpinnings of existing virtual reality educational applications or biosensor real-time affective analytics in mixed reality for medical education-based serious games in [32] and [83] are examples of evolving the learning process on educational platform-based applications. John et al. [47] created the VIRTUE virtual environment for stroke patients' cognitive rehabilitation. A study at the Countess of Chester Hospital enlisted forty volunteers to participate in virtual reality therapy. The MoCA test revealed early signs of improved mental recovery and a shorter stay in the hospital. Other applications and studies addressed in this study differ in their capacity to discern and identify distinct symptoms for patients with anxiety, depression, and stress using VR and EDA. In present studies, there is minimal agreement on the usefulness of VR and EDA applications and no recommended measurement of improvement for user accomplishment.

The Oculus Quest 2 and HP Reverb G2 are new and not applied in clinical trials, but they are not the only equipment accessible for research. As a result, the equipment's usability, comprehension, sensitivity to aural and visual cues, and cognitive control were all compromised. Communication, memory and recall, planning, navigation, prompting for activity execution, learning and research are critical areas to focus on in terms of development. An application to monitor and record MCI-related symptoms could be one of the possible development options. A notification system, for example, might be used to alert and help with medication or other everyday duties. On the other hand, the expense may discourage people from getting the help they need. Google Cardboard and Oculus Rift S, released in 2019 and 2013, are less expensive options. It's interesting looking into the simulations and user experience, although they're not quite as good as Oculus Quest and Quest 2. When communication and navigational challenges occur in people with MCI symptoms, one way is to help them. R.Patient et al [96]. are working on a prototype application that links assistance and activity awareness using machine learning methodologies. Anxiety, tension, and depression are all induced by MCI; nevertheless, interacting with VR and EDA while gathering activity can help people with MCI [36], [81], and [88].

The Alzheimer's Association and ADNI's material has restrictions through online access by participant data due to the limited dataset and evaluation questions. As a result, future research must continue to expand the dataset to obtain sufficient data on changes in MCI symptoms in persons. Second, merging VR with EDA when recognising MCI indications is challenging and complex. Furthermore, participants may be suffering from a range of health problems that are not wholly full disclosure, resulting in similar effects. Despite these encouraging results, many questions can find answers by comparing studies conducted with the same approach and equipment. There are, however, challenges because technology is continually improving. According to [18], a data set from ADNI is obtainable by using machine learning techniques to execute a framework and testing the application with non-MCI and MCI participants.

The Oculus Quest 2 and Fitbit, built for simulation and activity and containing the VR and EDA information, might be used to create a new creative data set, as demonstrated by the study in [45]. The VR and EDA would be coupled during training and recovery in this arrangement. MCI-related problems would be documented, observed, and labelled for data analysis [78]. COVID-19 makes it challenging to conduct such trials and recruit vulnerable subjects with MCI symptoms, as mentioned in [83]. Previous research by Goodman-Casanova et al. and Hampshire et al. looked into cognitive deficiencies, COVID-19, and confinement issues in older people with MCI or dementia [3], [36]. Future research should address the limitations and weaknesses of the proposed articles examined in this SLR. To begin with, the restricted data in the dataset used in this evaluation could represent a flaw in the paper. However, findings were identifiable from the data in line with earlier studies.

The validation and framework of the application of VR and EDA have been the main research focus from the reviewed articles. Table IV shows that involving VR and EDA in the review reduced duplicate searches by $18-40 \%$ (as recommended by PRISMA and Cochrane guidelines) [39], [40]. Despite PRISMA and The Cochrane Standards, the literature search for various methodologies in current systematic reviews was uneven, as shown in Figure 1. Other disorders, such as anxiety, stress, and depression, are identified with a high systematic literature review output. Examining VR and EDA to help with MCI broadens the scope of research. There has been a noticeable increase in interest in using VR and EDA to help people with MCI and similar conditions (Alzheimer's, Dementia, Parkinson's). Furthermore, determining the overall reliability of results, such as what was being measured and what technology was obtaining results with, was difficult due to the environment and differences in disorders such as human emotion, dementia, specific learning disorders, COVID-19, and maternal mental health, [36], [48], [51], [92]. The articles in this systematic review covered a wide range of topics. Covid-19 and applied to human emotion, dementia, specific learning problems, and maternal mental health 36], [48], [51], [92], as a consequence of many studies and contexts of the application and challenges caused by Covid19. For example, Chandler et al., Parong et al.[64], and Belleville et al. [71] applied a mixture of participants as a typical sample population shown in Figure 3 [20], [64], [71]. Participants differed broadly in age, experience, and health. The nature of MCI symptoms and equipment used may also affect the study outcomes shown in Table VIII. Chandler et al.[20], Belleville [71] did not further investigate alternatives in hardware that the latest technology could provide for user engagement, such as hand tracking and interacting with avatars and virtual objects [20], [71]. Whereas, Parong et al. [64] investigated the Cave Automated Virtual Environment (CAVE) and the Head-Mounted Display (HMD). Out of 91 journals, 44 studies with 18198 participants had a risk difference of $95 \%$ with an effect estimate of 2.25. A further 26 used 8858 
participants had a ratio of $95 \%$ with an effect estimate of 6.10 . Two studies involved VR and EDA using 91 participants [32], [83].

Therefore, preliminary evidence of VR and EDA being applied in cohort studies exploring Pedagogical Foundations of Existing Virtual Reality Educational Applications and Biosensor Real-Time Affective Analytics in Virtual and Mixed Reality Medical Education Serious Games. For the comparative scientific reasoning of results, sample sizes ranging from 3 to 1615 participants were discovered in the evaluated studies [20], [38]. Because there is no cross-comparison of results or reason, the small number of participants in studies is troublesome and could make the validity and research concepts vulnerable. Increasing data appropriateness, quantitative health datasets must be more clear about assessments. Memory loss, navigational and linguistic issues, and the ethical considerations of conducting experimental research, in general, are already confined to technology for cognitive help [4].

The Std. Mean Difference was also $95 \%$, but the effect estimate was improved to 0.60 , in Figure 3 . Thus, there is a vast shortage of research exploring VR and EDA for supporting MCI and other mental health conditions. Figure 2. The 93-th percentile is associated with the Z-Score $-\mathrm{z}=1.46$ was $92.79 \%$. Figure 3 leans more toward non-cognitive studies, whereas the funnel plot in Figure 4 is relatively symmetrical. Many studies fall outside the triangle (for example [28], [51], [88]), indicating heterogeneity in the strength of the association between cognitive and non-cognitive was also present in studies. Though, measurements of physical functions and emotions applied in some studies were only used as safeguard benchmarks instead of outcome measures shown in Table VI. These findings will doubtlessly be much scrutinised, but there are some immediately reliable conclusions to be made; there is a lack of in-depth research into the complexities of MCI involving VR and EDA.

The development of software is another factor contributing to the concept of a VR and EDA application for a scalable realtime application for the world's population, research, and medical field. Goodman-Casanova et al. and Hampshire et al. previously investigated cognitive deficits, COVID-19, and confinement issues in older persons with MCI or Dementia [3], [36]. The research we focused on was the Application of Virtual Reality and Electrodermal Activity for the Detection of Cognitive Impairment. It was necessary to wait until COVID19 restrictions lifted to allow for improved methodology and study set-up for data collection and 30 participants to be acceptable [96]. Whilst the research we focused on was the Application of Virtual Reality and Electrodermal Activity for the Detection of Cognitive Impairment, it was necessary to wait until COVID-19 restrictions were updated and lifted to allow for better methodology and study set-up data collection, as well as 30 participants to be acceptable. The ADNI data set discovered through the systematic review will train the framework and machine learning technique. Investigate machine learning strategies in MCI-related difficulties such as anxiety, stress, and depression to assist people.

\section{CONCLUSION \& FUTURE WORK}

In this article, a systematic review was performed to discover and map out the requirements for VR and EDA to support MCI. A framework applying three main concepts (VR, EDA, and ML) was suggested to identify the conditions for a real-time system that could be scalable to the world's population with the increase of MCI whilst applying an agile methodology. Based on the systematic review of literature results, it is established that combining VR, EDA, and ML is currently less researched than EEG with VR and ML, which has a broader selection of articles to analyse from the past decade. An increase of research is required for the purpose of data collection for VR and EDA results to be able to improve efficient models.

On the other hand, there has been an increase in the literature regarding ML EEG and EDA within the scientific field for clinical trials and validation purposes. But, MCI assessments are still generic and are not specific to any one condition, making diagnosis harder than improving a user's health. This is explained by assessment levels set out by the medical field in their literature. The research on the development of legal, ethical, and minimal standards is a significant consideration when validating because of the human element. Finally, we have identified a new research path for the application of VR, EDA, and ML. This article was looking to understand the key concepts and links between MCI, symptoms, and how technology can enhance users' lives to reach their potential. The research and medical field have connections that could support the development of VR, EDA, and ML frameworks to improve individuals' health regardless of condition.

\section{REFERENCES}

[1] D. W. Shankle, Causes of Cognitive Impairment, 2019 https://www.hoag.org/specialitiesservices/neurosciences/programs/memoryco gnitive-disorders/types-of-memory-cognitive

[2] K. Cosic' et al., "Artificial intelligence in the prediction of mental health disorders induced by the COVID-19 pandemic among health care workers," Croatian Medical Journal, vol. 61, no. 3, pp. 279-288, 2020.

[3] J. Goodman-Casanova et al., "Telehealth home support during COVID-19 confinement for community-dwelling older adults with mild cognitive impairment or mild dementia: survey study," 2018. https://www.jmir.org/2020/5/e19434/

[4] R. Singh et al., "Significant virtual reality applications for COVID-19 pandemic," 2020._https://doi.org/10.1016/j.dsx.2020.05.011

[5] M.Hopkinson et al., "Cognitive behavioural therapy for depression, anxiety, and stress in caregivers of dementia patients: A systematic review and meta-analysis," 2019. doi.org/10.1093/geront/gnx217

[6] S. Swayamsiddha et al., Application of cognitive Internet of Medical Things for COVID-19 pandemic, 2020. https://doi.org/10.1016/ j.dsx.2020.06.014

[7]E. Prather et al., "A systematic literature review on dynamic cognitive augmentation through immersive reality: challenges and perspectives," SPIE.DigitalLibrary, 2020.

[8] S. Wakefield et al., "The effectiveness of brief cognitive analytic therapy for anxiety and depression: A quasi-experimental case-control study," British Journal of Clinical Psychology, 2021. 10.1111/bjc.12278

[9] L. Cook et al., "Reducing stress and preventing depression (RESPOND): randomised controlled trial of web-based rumination-focused cognitive behavioural therapy," 2019. 10.2196/11349

[10] A. Yohannes et al., "Association of mild cognitive impairment and characteristic of COPD and overall health status in a cohort study," Expert Review of Respiratory Medicine, 2020.

$10.1080 / 17476348.2021 .1838278$ 
[11] Y. Liu et al., "A Review of the Application of Virtual Reality Technology in the Diagnosis and Treatment of Cognitive Impairment," Frontiers in Aging Neuroscience, vol. 11, 2019. 10.3389/fnagi.2019.00280 [12] F. Ihmig et al., "Online anxiety level detection from biosignals: Machine learning based on a randomised controlled trial with spider-fearful individuals," PLoS ONE, vol. 15, no. 6, pp. 1-20, 2020.

[13] S. Glegg et al., "Barriers, Facilitators and Interventions to Support Virtual Reality Implementation in Rehabilitation: A Scoping Review," vol. 10, 2018._10.1016/j.pmrj.2018.07.004

[14] R. Eijlers et al., Systematic Review and Meta-analysis of Virtual Reality in Pediatrics: Effects on Pain and Anxiety," ncbi.nlm.nih.gov, 2019.

10.1213/ANE. 000000000004165

[15] D. Wickramasuriya et al., A novel filter for tracking real-world cognitive stress using multi-time-scale point process observations," ieeexplore.ieee.org, 2019. 10.1109/EMBC.2019.8857917

[16] H. Posada-Quintero et al., "Electrodermal activity is sensitive to cognitive stress underwater," Frontiers in Physiology, vol. 8, 2018. 10.3389/fphys.2017.01128

[17] N. Anderson et al., "State of the science on mild cognitive impairment (MCI)," cambridge.org, 2019. 0.1017/S1092852918001347

[18] S. Weech et al., "Presence and cybersickness in virtual reality are negatively related: A review," Frontiers in Psychology, vol. 10, 2019. 10.3389/fpsyg.2019.00158

[19] D. Moss, "Biofeedback-Assisted Relaxation Training: A Clinically Effective Treatment Protocol," Biofeedback-Assisted Relaxation Training: A Clinically Effective Treatment Protocol, vol. 48, pp.32-40, 2020. www.aapb.org

[20] M. Chandler et al., "Comparative effectiveness of behavioural interventions on quality of life for older adults with mild cognitive impairment: a randomised clinical trial,"_2019.

10.1001/jamanetworkopen.2019.3016

[21] E. C. Schizoph et al., "The effect of loneliness on depression: A metaanalysis," International Journal of Social Psychiatry, vol. 64, no. 5, pp. 427435, 2018. 10.1177/0020764018776349

[22] T. Rose et al., "Immersion of virtual reality for rehabilitation-Review," 2018. https://doi.org/10.1016/j.apergo.2018.01.009

[23] A. Souza et al., "An automated functional testing approach for virtual reality applications," Software Testing Verification and Reliability, vol. 28, 2018. $10.1002 /$ stvr. 1690

[24] M. Peskin et al., "The relationship between post-traumatic and depressive symptoms during virtual reality exposure therapy with a cognitive enhancer," 2019. 10.1016/j.janxdis.2018.03.001

[25] O. Balan et al., "Sensors system methodology for artefacts identification in Virtual Reality games," 2019 International Symposium on Advanced Electrical and Communication Technologies, 2019.

10.1109ISAECT47714.2019.9069719

[26] S. Campanella et al., "Special Report on the Impact of the COVID-19 Pandemic on Clinical EEG and Research and Consensus Recommendations for the Safe Use of EEG," 2020. 10.1177/1550059420954054

[27] Kamińska' et al., "Stress Reduction Using Bilateral Stimulation in

Virtual Reality," IEEE Access, vol. 8, 2020.

doi.org/10.1109/ACCESS.2020.3035540

[28] M. Browning et al., "Can Simulated Nature Support Mental Health? Comparing Short, Single-Doses of 360-Degree Nature Videos in Virtual

Reality With the Outdoors," Frontiers in Psychology, vol. 10, 2020

10.3389/fpsyg.2019.02667

[29] J. Lyu et al., "Gender-specific associations of sensory impairments with depression and cognitive impairment in later life," ncbi.nlm.nih.gov, 2019.

https://dx.doi.org/10.30773/pi.2018.06.28.2

[30] A. Dzedzickis et al., "Human Emotion Recognition: Review of sensors and methods," Sensors (Switzerland), vol. 20, no. 3, 2020.

$10.3390 / \mathrm{s} 20030592$

[31] H. Kim et al., "Recent Advances in Wearable Sensors and Integrated Functional Devices for Virtual and Augmented Reality Applications,

"Advanced Functional Materials, 2020. 10.1002/adfm.202005692

[32] E. Johnston et al., "Exploring Pedagogical Foundations of Existing

Virtual Reality Educational Applications: A Content Analysis Study, "Journal of Educational Technology Systems, vol. 46, no. 4, pp.414-439, 2018.

$10.21767 / 2574-2825.100030$
[33] Hsieh et al., "Preliminary Study of VR and AR Applications in Medical and Healthcare Education," Journal of Nursing and Health Studies, 2018.

$[10.1177 / 0047239517745560$

[34] N. Forand et al., "Efficacy of guided iCBT for depression and mediation of change by cognitive skill acquisition", 2018.

https://doi.org/10.1016/j.beth.2017.04.004

[35] S. Kengne et al., "action-centred Exposure Therapy (ACET): A New

Approach to the Use of Virtual Reality to the Care of People with Post-

Traumatic Stress Disorder," 2018. 10.3390/ bs8080076

[36] A. Hampshire et al., "Cognitive deficits in people who have recovered from COVID-19 relative to controls: An N=84,285 online study, pp. 2020

2020. $10.1101 / 2020.10 .20 .20215863$

[37] K. Kyriakou et al., "Detecting moments of stress from measurements of wearable physiological sensors", 2019. https://doi.org/10.3390/s19173805 [38] S. Jungmann et al., "Health anxiety, cyberchondria, and coping in the current COVID-19 pandemic: Which factors are related to coronavirus anxiety?", 2020. https://doi.org/10.1016/j.janxdis.2020.102239

[39] S. Mrakic-Sposta et al., "Effects of combined physical and cognitive virtual reality-based training on cognitive impairment and oxidative stress in MCI patients: A pilot study," Frontiers in Aging Neuroscience, vol. 10, 2018. 10.3389/fnagi.2018.00282

[40] T. Rodrigues et al., "A Quality of Experience assessment of haptic and augmented reality feedback modalities in a gait analysis system," PLoS ONE, vol. 15, no. 3, pp. 1-19, 2020. 10.1371/

journal.pone. 0230570

[41] C. Lin et al., "Impact of virtual reality (VR) experience on older adults' well-being," 2018. https://link.springer.com/ chapter/10.1007/978-3-31992037-5_8

[42] J. Gold et al., "Is virtual reality ready for prime time in the medical space? A randomised control trial of pediatric virtual reality for acute procedural pain management", 2018. https:// doi.org/10.1093/jpepsy/jsx129 [43] W. Aslam et al., "A systematic review of the diagnostic accuracy of automated tests for cognitive impairment," Wiley Online Library, vol. 33, no. 4, pp. 561-575, 2018. 10.1002/gps.4852

[44] A. Society, "Mild cognitive impairment (MCI)," 2020 https://www.alzheimers.org.uk/about-dementia/types-dementia/mildcognitive-impairment-mci

[45] PRISMA, "PRISMA," 2021. http://www.prisma

[46] C. Training, "Cochrane Training and Revman," 2021.

https://training.cochrane.org/online-learning/core-softwarerevman/revman-5download

[47] N. W. John et al., "Virtual Reality Environment for the Cognitive Rehabilitation of Stroke Patients", 2019. https://

ieexplore.ieee.org/stamp/stamp.jsp?tp=\&arnumber $=8864513$

[48] D. Stamate et al., "A Machine Learning Framework for Predicting Dementia and Mild Cognitive Impairment," ieeexplore.ieee.org, 2019. https://doi.org/10.1109/ICMLA.2018.00107

[49] J. Radianti et al., "A systematic review of immersive virtual reality applications for higher education: Design elements, lessons learned, and research agenda", vol. 147, pp. 103 778-103 778, 2019. 10.1016/ j.compedu. 2019.103778

[50] P. Bernardo et al., "Mood Induction Using Virtual Reality: a Systematic Review of Recent Findings," Journal of Technology in Behavioral Science, pp. 1-22, 2020. https://doi.org/10.1007/s41347-02000152-9

[51] C. Rico-Olarte et al., "Towards Classifying Cognitive Performance by Sensing Electrodermal Activity in Children with Specific Learning Disorders,"

IEEE Access, 2020. 10.1109/ACCESS.2020.3033769

[52] S. Wang et al., "Cognitive behavioural therapy for post-stroke depression: a meta-analysis", 2018. 10.1016/ j.jad.2018.04.011

[53] R. Sánchez-Reolid et al., "Machine Learning for Stress Detection from Electrodermal Activity: A Scoping Review," Preprints, 2020.

10.20944/preprints202011.0043.vl

[54] J. Marín-Morales et al., "Emotion recognition in immersive virtual reality: From statistics to affective computing," Sensors (Switzerland), vol. 20, no. 18 , pp. 1-26, 2020. 10.3390/s2018516

[55] C. Soumya et al., "Affect Recognition using Psychophysiological Correlates in High-Intensity VR Exergaming," Conference on Human Factors 
[76] S. Spasov et al., "A parameter-efficient deep learning approach to predict conversion from mild cognitive impairment to Alzheimer's disease," 2019

https://doi.org/10.1016/j.neuroimage.2019.01.031

K. Fadeev et al., "Too Real to Be Virtual: Autonomic and EEG Responses to Extreme Stress Scenarios in Virtual Reality," Behavioural Neurology, 2020. $10.1155 / 2020 / 5758038$

[77] L. Fodor et al., "The effectiveness of virtual reality-based interventions for symptoms of anxiety and depression: A meta-analysis," 2018.

10.1038/s41598-018-28113-6

[78] Fertleman et al., "A discussion of virtual reality as a new tool for training healthcare professionals," Frontiers in Public Health, vol. 6, 2018. $0.3389 /$ fpubh.2018.00044

[80] R. Slot et al., "Subjective Cognitive Impairment Cohort (SCIENCe): Study design and first results," Research and Therapy, vol. 10, no. 1, 2018. 10.1186/s13195-018-0390-y

[81] M. Zetterberg et al., "Internet-based cognitive behavioural therapy of perfectionism: comparing regular therapist support and support upon request," 2019. 10.1016/j.invent.2019.02.001

[82] Z. Yang et al., "Cognitive behavioural therapy is associated with enhanced cognitive control network activity in major depression and posttraumatic stress disorder," 2018. https://doi.org/10.1016/ j.bpsc.2017.12.006 [83] A. Panagiotis et al., "Biosensor Real-Time Affective Analytics in Virtual and Mixed Reality Medical Education Serious Games: Cohort Study," JMIR Serious Games, vol. 8, no. 3, 2020. 10.2196/17823

[84] S. Stjerneklar et al., "Internet-based cognitive behavioural therapy for adolescents with anxiety disorders: A feasibility study," Internet-based cognitive behavioural therapy for adolescents with anxiety disorders: A feasibility study, 2018. https://doi.org/10.1016/ j.invent.2018.01.001 [85] F. Pieruccini-Faria et al., "Mapping Associations Between Gait Decline and Fall Risk in Mild Cognitive Impairment," Wiley Online Library, vol. 68, no. 3, pp. 576-584, 2019. 10.1111/jgs. 16265

[86] T. Donker et al., "Effectiveness of self-guided app-based virtual reality cognitive behaviour therapy for acrophobia: a randomised clinical trial,"

JAMA Network, 2019. https://

pubmed.ncbi.nlm.nih.gov/30892564/

[87] F. Norlund et al., "Internet-based cognitive behavioural therapy for symptoms of depression and anxiety among patients with a recent myocardial infarction: the U-CARE heart," 2018. 10.2196/jmir.9710 [88] W. Aslam et al., "A systematic review of the diagnostic accuracy of automated tests for cognitive impairment," Wiley Online Library, vol. 33, no. 4 , pp. 561-575, 2018. 10.1002/gps. 4852

[89] J. Lin et al., "Assessing the influence of repeated exposures and mental stress on human wayfinding performance in indoor environments using virtual reality technology," Assessing the influence of repeated exposures and mental stress on human wayfinding performance in indoor environments using virtual reality technology, 2019.

https://doi.org/10.1016/j.aei.2018.11.007

[90] F. Salamone et al., "Correlation between indoor environmental data and biometric parameters for the impact assessment of a living wall in a ZEB lab," Sensors (Switzerland), vol. 20, no. 9, 2020. 10.3390/s20092523 [91] M. Martinez et al., "Physiological assessment of User eXprience supported by Immersive Environments: First input from a literature review," ConVRgence (VRIC) Virtual Reality International Conference, pp. 89-108, 2020. https://hal.archives-ouvertes.fr/

hal-02551341

[92] Davenport et al., "Moms Are Not OK: COVID-19 and Maternal Mental Health," Global Women's Health, vol. 1,

2020.10.3389/fgwh.2020.00001

[93] A. Jahangir et al., "Physiological Implications of Mental Walkthrough

Social Engagement," 2020. https://www.researchgate.net [94] S. Ge et al., "Technology-based cognitive training and rehabilitation interventions for individuals with mild cognitive impairment: A systematic review," BMC Geriatrics, vol. 18, no. 1, 2018. 10.1186/s12877-018-0893-1 [95] B. Shulman et al., "Feasibility of a mindfulness-based cognitive therapy group intervention as an adjunctive treatment for postpartum depression and anxiety," 2018. https://doi.org/10.1016/j.jad.2017.12.065 [96] R. Patient et al., "Application of Virtual Reality and Electrodermal Activity for the Detection of Cognitive Impairment", DeSE2021, 2021. 